\title{
Hypothesis-generating study on the effect of the ACLS guidelines on the use of atropine in cardiac arrest at a community hospital
}

This article was published in the following Dove Press journal: Research Reports in Clinical Cardiology

\author{
Matthew Mo Kin Kwok ${ }^{1,2}$ \\ Rob Stenstrom' \\ Edward Mak ${ }^{2}$ \\ Ka Wai Cheung'
}

'Department of Emergency Medicine, Faculty of Medicine, University of British Columbia, Vancouver, BC, Canada; ${ }^{2}$ Emergency Department, Richmond Hospital, Vancouver Coastal Health, Richmond, BC, Canada

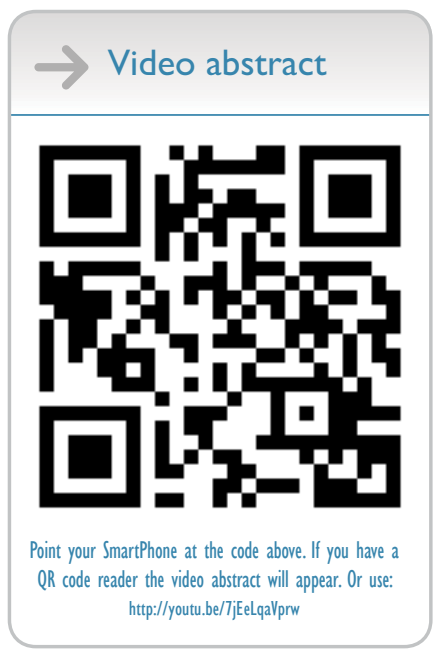

Correspondence: Matthew Mo Kin Kwok Emergency Department, Richmond Hospital, 7000 Westminster Hwy, Richmond, BC, V6X IA2, Canada $\mathrm{Tel}+\mathrm{I} 6048128162$

Fax + I 6044474353

Email matthew.kwok@vch.ca
Background: Barriers exist in translating clinical practice guidelines into medical management of patients. These barriers result in delay in translating the Advanced Cardiac Life Support (ACLS) guidelines into clinical practice. We conducted a pilot study employing the recommendation change in atropine usage in the 2010 ACLS guideline algorithm to examine the time lag in translating guidelines into medical practice.

Methods and results: We completed a retrospective chart review at a community hospital. Study data was derived from cardiac arrest records from the emergency department between January 1, 2009 and December 31, 2013, before and after the publication of the 2010 ACLS guidelines. All cardiac arrests in the form of asystole and/or pulseless electrical activity at some time during resuscitation in patients aged 19 years and older were included in the study. We examined whether atropine was used during the resuscitation. We studied the use of epinephrine as a control. A time versus atropine and a time versus epinephrine usage graphs were generated and examined. Fifty-five resuscitations met inclusion criteria. Although the 2010 ACLS guidelines were first presented in October 2010, we observed that change in atropine use occurred around the summer of 2011. There was no change in the use of epinephrine.

Conclusion: Despite several guideline dissemination strategies, a time lag was found in physicians' adaptation of the ACLS guidelines.

Keywords: cardiopulmonary resuscitations, resuscitations, Advanced Cardiac Life Support, guidelines translation, guidelines dissemination

\section{Background}

The purpose of clinical practice guidelines is to guide physicians in making appropriate decisions in specific clinical circumstances. Their successful implementation should translate research into effective practices and thus improve patient outcomes. ${ }^{1}$ In particular, the American Heart Association has introduced the Advanced Cardiac Life Support (ACLS) guidelines to improve cardiac arrest quality of care. ${ }^{2}$ Physicians have to be aware of and utilize these guidelines in order to benefit patients and improve outcomes.

The American Heart Association introduced the ACLS guidelines in 2010. In this update, atropine was removed from the asystole and pulseless electrical activity (PEA) cardiac arrest algorithms. ${ }^{3}$ Several potential barriers have been identified in physicians' uptake of the ACLS guidelines. ${ }^{3}$ These barriers result in delay in translating ACLS guidelines into clinical practice. ${ }^{4-6}$ The time delay in physicians' uptake of ACLS guidelines has not been examined, and the time lag in changing medical management after 
the publication of the guidelines has not been quantified. We conducted a study employing the recommendation change in atropine usage in the ACLS guideline algorithm to examine the time lag in translating guidelines into medical practice.

\section{Methods}

We conducted a retrospective chart review at a community hospital after receiving ethics approval from the University of British Columbia Clinical Research Ethics Board. As this is a retrospective observational study meeting the institutional minimal risk standard, consent was not obtained. Study data was derived from cardiac arrest records between January 1, 2009 and December 31, 2013, before and after the publication of the 2010 ACLS guidelines and recorded in an encrypted secured password-protected database. All cardiac arrests in the form of asystole and/or PEA at some time during resuscitation in patients aged 19 years and older were included in the study. This included all prehospital patients presenting to the emergency department and in-hospital emergency department patients with asystole and PEA cardiac arrests. Patients were excluded if they were younger than 19 years of age, the cardiac arrest was not in the form of asystole or PEA, or the patient had a "do not resuscitate" order. For each identified patient, the patient's age, gender, date of arrest, type(s) of arrest, prehospital vs in-hospital arrest, initials of the emergency physician resuscitation leader, and the medications given during the resuscitation were recorded. We examined whether atropine was used during the resuscitation. We also studied the use of epinephrine as a control. A time versus atropine and a time versus epinephrine usage graph were generated and examined.

\section{Results}

Between January 1, 2009 and December 31, 2013, 64 cardiac arrest records were found. In total, 55 resuscitations met the inclusion criteria. This included 38 prehospital and 17 inhospital emergency department asystole and/or PEA cardiac arrests. Seventeen emergency physicians were identified to be involved with the resuscitations. The median age of the cardiac arrest patients was 72 . There were 22 women and 33 men. Atropine was used in 21 cardiac arrests, while epinephrine was used in 51 cardiac arrests. A time vs atropine use graph and a time vs epinephrine use graph were generated (Figures 1 and 2).

\section{Discussion}

We conducted a hypothesis-generating study on the delay in physicians' adaptation of clinical practice guidelines by exam-

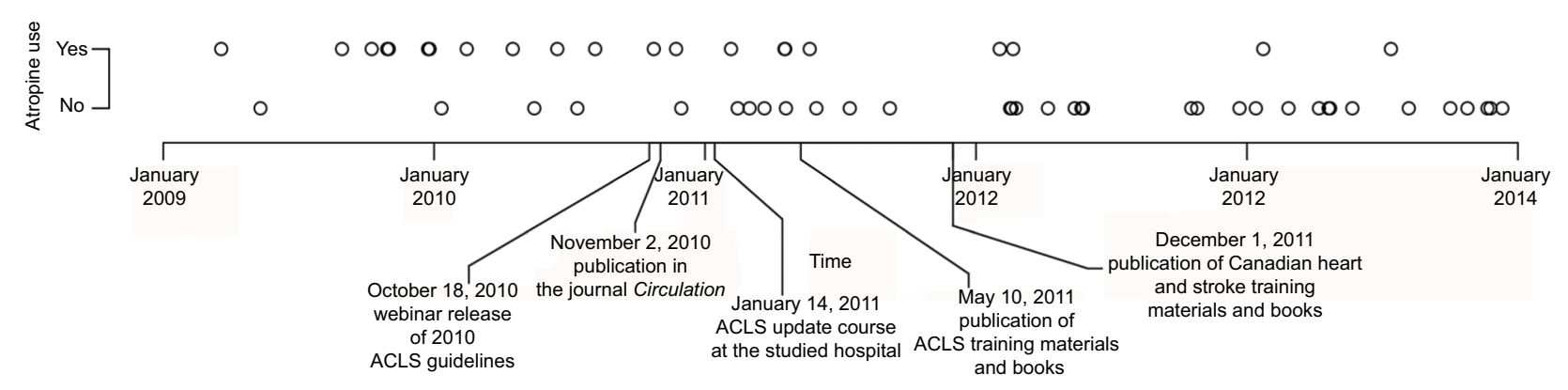

Figure I Atropine use in asystole and/or PEA cardiac arrest.

Abbreviations: PEA, pulseless electric activity; ACLS, Advanced Cardiac Life Support.

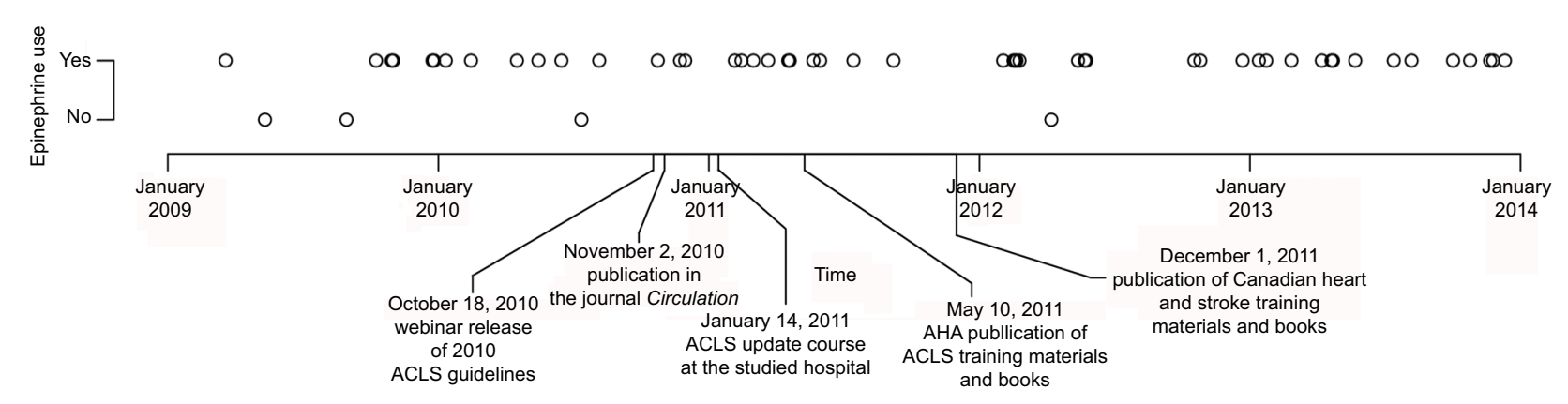

Figure 2 Epinephrine use in asystole and/or PEA cardiac arrest.

Abbreviations: PEA, pulseless electric activity; ACLS, Advanced Cardiac Life Support. 
Box I The timeline of the 2010 ACLS guidelines dissemination

- October 18, 2010: Webinar release of 2010 ACLS guidelines

- November 2, 2010: Publication in the journal circulation

- January 14, 201 I: ACLS update course at the studied hospital

- May 10, 20II: AHA publication of ACLS training materials and books

- December I, 20I I: Publication of Canadian heart and stroke ACLS training materials and books

Abbreviation: ACLS, Advanced Cardiac Life Support.

ining the effect of the 2010 ACLS guidelines on the use of atropine in asystole/PEA cardiac arrests. Although the ACLS guidelines were first presented in October 2010 (Box 1), we observed that change in atropine use occurred later around the summer of 2011. These findings elicit 2 questions. First, what guideline dissemination strategies can be used to decrease the lag time in translating ACLS guidelines into practice? Second, what should the timing of these dissemination strategies be to maximize its effect? We believe through quantifying the time lag in translating guidelines into practice, we can better answer these questions by studying whether changes in the type and timing of guideline dissemination strategies will decrease this lag time in the future.

\section{Disclosure}

The authors report no conflicts of interest in this work.

\section{References}

1. Cabana MD, Rand CS, Powe NR, et al. Why don't physicians follow clinical practice guidelines? JAMA. 1999;282(15):1458-1465.

2. Carveth S. Standards for cardiopulmonary resuscitation and emergency cardiac care. JAMA. 1974;227(7):796-797.

3. Field JM, Hazinski MF, Sayre MR, et al. 2010 American Heart Association guidelines for cardiopulmonary resuscitation and emergency cardiovascular care. Circulation. 2010;122(Suppl 3):S640-S946.

4. Weingarten S. Translating practice guidelines into patient care. Chest. 2000;118(Suppl 2):4S-7S.

5. Bigham BL, Koprowicz K, Aufderheide TP, et al. Delayed prehospital implementation of the 2005 American Heart Association guidelines for cardiopulmonary resuscitation and emergency cardiac care. Prehosp Emerg Care. 2010;14(3):355-360.

6. Govindarajan P, Lin L, Landman A, et al. Practice variability among the EMS systems participating in Cardiac Arrest Registry to Enhance Survival (CARES). Resuscitation. 2012;83(1):76-80.
Research Reports in Clinical Cardiology

\section{Publish your work in this journal}

Research Reports in Clinical Cardiology is an international, peerreviewed, open access journal publishing original research, reports, editorials, reviews and commentaries on all areas of cardiology in the clinic and laboratory. The manuscript management system is completely online and includes a very quick and fair peer-review system.

\section{Dovepress}

Visit http://www.dovepress.com/testimonials.php to read real quotes from published authors. 